\title{
Dielectric Behavior of Poly(methyl methacrylate)s in Dilute Solutions
}

\author{
Yasuo Iwasa, Satoru Mashimo, ${ }^{*}$ and Akio Chiba \\ Department of Applied Physics, School of Science and Engineering, \\ Waseda University, Shinjuku-ku, Tokyo, Japan.
}

(Received January 19, 1976)

\begin{abstract}
Dielectric measurements were carried out on dilute solutions of isotactic and syndiotactic poly(methyl methacrylate)s in toluene and dioxane in the frequency range of $1-150 \mathrm{MHz}$ over a wide range of temperature. A symmetrical dielectric loss curve was observed for each solution. The dielectric relaxation behavior of these two types of poly(methyl methacrylate)s was not much affected by the solvent viscosity. It is suggested that the dipole attached to a flexible side-chain performs a frictionless motion across a single potential barrier arising from intramolecular interactions between chain segments. The relaxation time calculated by using the Kramers rate constant for small friction is in close agreement with that observed. This agreement indicates that the motional unit involved in the relaxation process is fairly small. It is shown that the distribution of relaxation times and its temperature dependence can be well interpreted in terms of the equation presented by Fröhlich.
\end{abstract}

KEY WORDS Dielectric Relaxation / Poly(methyl methacrylate) / Stereoregularity / Dilute Solution / Solvent Viscosity / Flexible Side-Chain / Intramolecular Interaction / Potential Barrier / Relaxation Time /

Poly(methyl methacrylate) (PMMA) is one of the polar polymers which have dipoles attached to flexible side-chains. Each dipole can rotate not only around the side-chain but also around the main-chain, ${ }^{1}$ so that one may expect to observe multiple dielectric relaxation processes reflecting different types of dipolar motions. In fact, it has been found that PMMA in the solid state exhibits two kinds of relaxation processes., ${ }^{2,3}$ In the case of dilute solution, nevertheless, only one kind of dielectric relaxation was observed, ${ }^{4-6}$ and it has been assumed that the side-chain sets up a cooperative motion with the main-chain..$^{4,5}$ However, no satisfactory explanation for this relaxation mechanism has been established yet.

The purpose of the present work is to investigate the dielectric relaxation mechanism of PMMA in dilute solution. It is important to examine the effect of the stereoregularity on the dielectric behavior of PMMA in solution, because the arrangement of dipoles in a polymer

* Present address: Department of Physics, Faculty of Science, Tokai University, Hiratsuka, Kanagawa, Japan. molecule is altered by the change in the stereoregularity or, in other words, by the change in the local conformation. It has been reported that dielectric properties (e.g., relaxation time, activation energy, molar polarization, and so on) of PMMA in dilute solutions are greatly affected by its stereoregularity. ${ }^{6-8}$ However, the experimental conditions, especially of the frequency, employed in these studies were limited within narrow ranges, and the data obtained were not extensive enough to meet the present purpose. Thus, our measurements were carried out on both isotactic and syndiotactic PMMA in the range of frequency from 1 to $150 \mathrm{MHz}$ and in that of temperature from -36 to $60^{\circ} \mathrm{C}$.

Recently, Mashimo, et al., reported that the dielectric behavior of atactic PMMA (A-PMMA) in solution ${ }^{5}$ is somewhat different from that of vinyl-type polymers such as poly ( $p$-chlorostyrene) $(\mathrm{P} p \mathrm{CS})$ in solution. ${ }^{9}$ They indicated that the observed dielectric relaxation process of the latter can be attributed to the main-chain motion and the solvent viscosity plays a dominant role in reorientating the dipole. ${ }^{9}$ On the other hand, 
Dielectric Behavior of Polymer in Solution

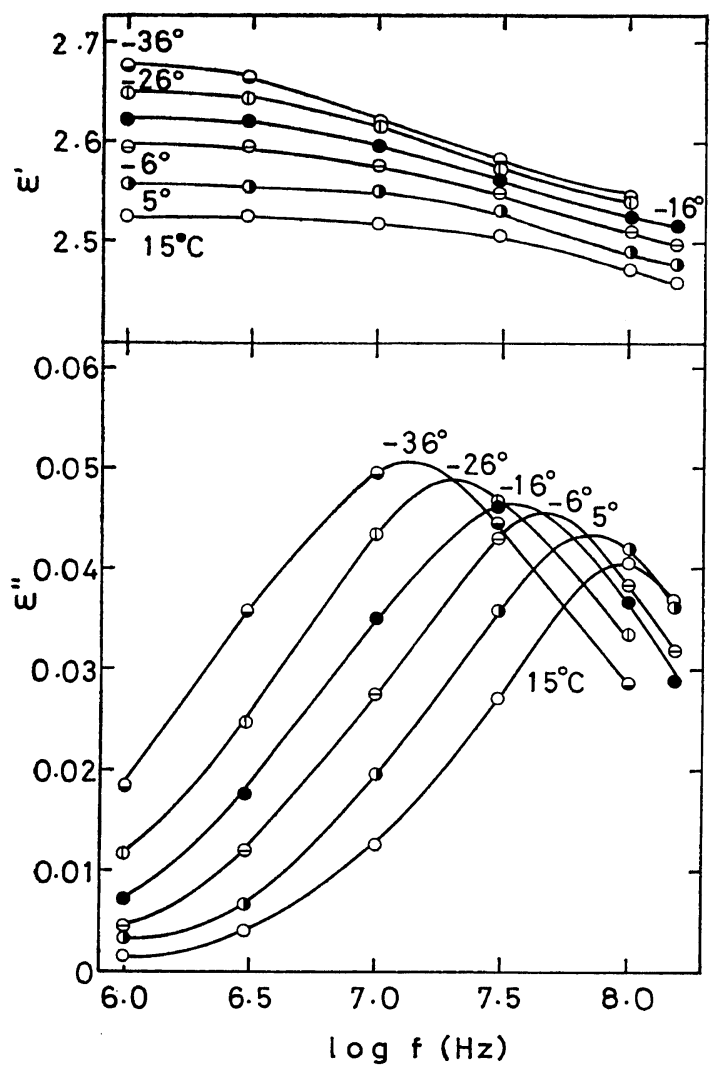

Figure 1. Frequency dependence of dielectric constant and loss at various temperatures for IPMMA in toluene, $4.99 \mathrm{wt} \%$.

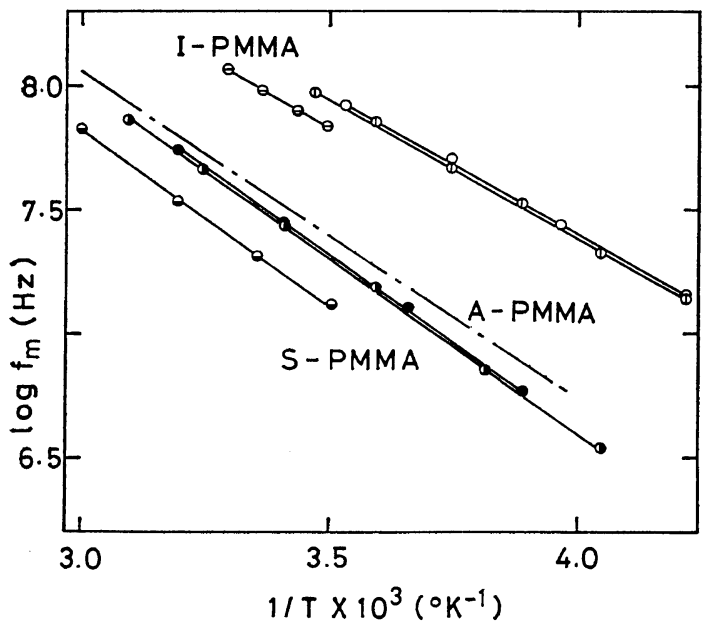

Figure 3. Plot of $\log f_{\mathrm{m}}$ against $1 / T: \quad 0,2.00 \mathrm{wt} \%$ I-PMMA in toluene; (1), $4.99 \mathrm{wt} \% ; \ominus, 2.02 \mathrm{wt} \%$ in dioxane; O, 2.04 wt $\%$ S-PMMA in toluene; $(1$, $4.90 \mathrm{wt} \% ; \Theta, 4.82 \mathrm{wt} \%$ in dioxane. The data for $5.40 \mathrm{wt} \%$ A-PMMA in toluene taken from ref 5 are added, represented by a chain line.

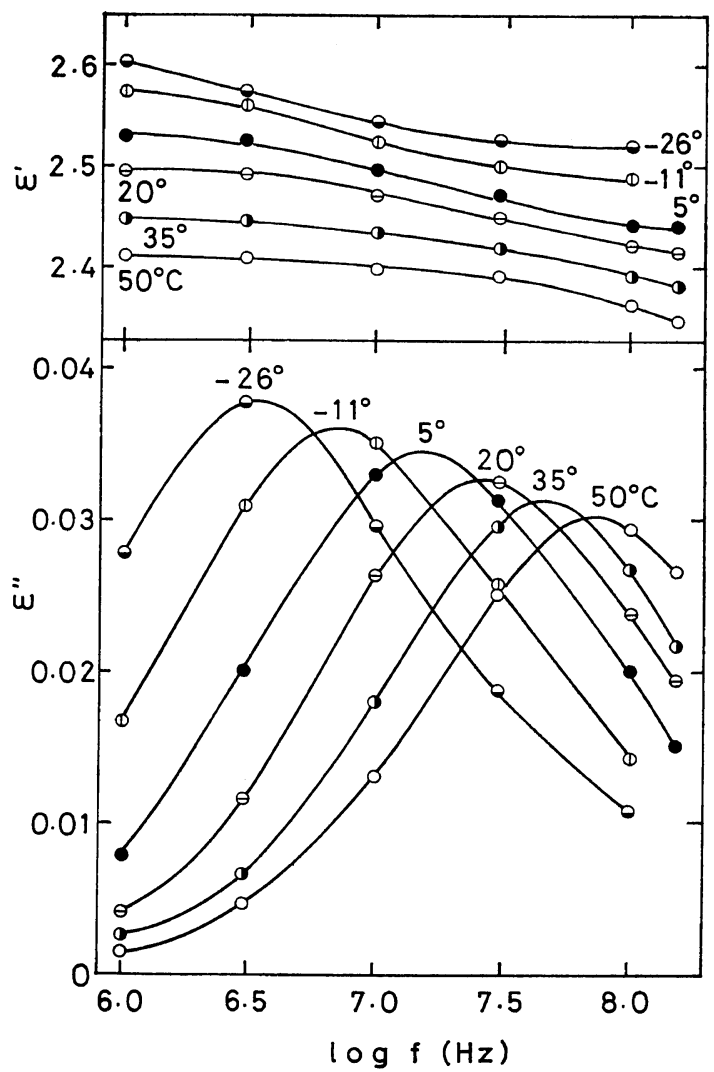

Figure 2. Frequency dependence of dielectric constant and loss at various temperatures for $\mathbf{S}$ PMMA in toluene, $4.90 \mathrm{wt} \%$.

Table II. Apparent activation energies for various PMMA solutions

\begin{tabular}{llcc}
\hline Polymer & Solvent & Concn, wt $\%$ & $\Delta H_{\mathrm{A}}, \mathrm{kcal} / \mathrm{mol}$ \\
\hline \multirow{3}{*}{ I-PMMA } & Toluene & 2.00 & 5.1 \\
& & 4.99 & 5.1 \\
& Dioxane & 2.02 & 5.3 \\
\hline \multirow{3}{*}{ S-PMMA } & Toluene & 3.04 & 6.5 \\
& & 4.90 & 6.5 \\
& Dioxane & 4.82 & 6.5
\end{tabular}

ture, as shown in Figure 3. In each case, this plot gives a straight line. The apparent activation energy $\Delta H_{\mathrm{A}}$ is determined from the slope of this line. The values of $\Delta H_{\mathrm{A}}$ for I- and SPMMA are presented in Table II.

In dilute solution, the values of $\Delta H_{\mathrm{A}}$ for each polymer in toluene and dioxane are approximately constant regardless of the solvent viscosity, though the activation energy for the 
Table III. Ratio of relaxation time to solvent viscosity

\begin{tabular}{lccccc}
\hline Polymer & Temp, ${ }^{\circ} \mathrm{C}$ & $\begin{array}{c}\left(\tau / \eta_{0}\right)_{\mathrm{T}} \times 10^{7}, \\
\text { sec poise }\end{array}$ & $\begin{array}{c}\left(\tau / \eta_{0}\right)_{\mathrm{D}} \times 10^{7}, \\
\text { sec poise }\end{array}$ & $\left(\tau / \eta_{0}\right)_{\mathrm{T}} / \overline{\left(\tau / \eta_{0}\right)}$ & $\left(\tau / \eta_{0}\right)_{\mathrm{D}} /\left(\tau / \eta_{0}\right)$ \\
\hline I-PMMA $^{\mathrm{a}}$ & 30 & 2.0 & 1.3 & 1.21 & 0.79 \\
S-PMMA $^{\mathrm{b}}$ & 13 & 2.8 & 1.6 & 1.27 & 0.73 \\
& 30 & 7.8 & 6.0 & 1.13 & 0.87 \\
PpCS & 13 & 12 & 8.5 & 1.17 & 0.83 \\
\hline
\end{tabular}

a Toluene, 4.99\%; dioxane, $2.02 \%$.

b Toluene, $4.90 \%$; dioxane, $4.82 \%$.

c Obtained from the data presented in ref 9.

solvent viscosity changes from $2.1 \mathrm{kcal} / \mathrm{mol}$ for toluene to $3.0 \mathrm{kcal} / \mathrm{mol}$ for dioxane. This feature shows a distinct contrast to that obtained for vinyl-type polymers, ${ }^{9}$ and resembles that obtained for A-PMMA. ${ }^{5}$

\section{Relaxation Time}

Recently, by employing the Kramers theory for large friction and the Stokes law, it was shown that, when the dielectric relaxation process reflects the segmental motion of the mainchain, the relaxation time is proportional to the solvent viscosity. ${ }^{9}$ In order to examine whether or not the relaxation time $\tau$ is affected by the solvent viscosity $\eta_{0}$, the ratio $\left(\tau / \eta_{0}\right)$ was evaluated for each solution. The values of $\tau$ at temperatures where measurements were not carried out were calculated by the use of $f_{\mathrm{m}}-1 / T$ relation.

The results are shown in Table III. Here, $\overline{\left(\tau / \eta_{0}\right)}$ represents the arithmetic mean value of $\left(\tau / \eta_{0}\right)_{\mathrm{T}}$ and $\left(\tau / \eta_{0}\right)_{\mathrm{D}}$, which is defined to estimate the relative error of the constancy of the ratio $\left(\tau / \eta_{0}\right)$. Here, subscripts $\mathrm{T}$ and $\mathrm{D}$ denote toluene and dioxane, respectively. The data on $\mathrm{P} p \mathrm{CS}$ reported by Mashimo and $\mathrm{Chiba}^{9}$ are quoted to compare with the present data. It is found that the values of $\left(\tau / \eta_{0}\right)_{\mathrm{T}}$ and $\left(\tau / \eta_{0}\right)_{\mathrm{D}}$ for both PMMA deviate from the mean value, while the value of $\left(\tau / \eta_{0}\right)_{\mathrm{T}}$ for $\mathrm{P} p \mathrm{CS}$ evaluated from ref. 9 is almost identical with that of $\left(\tau / \eta_{0}\right)_{D}$. This fact means that in the cases of both I- and S-PMMA in dilute solution, the relaxation time is not proportional to the solvent viscosity.

\section{Magnitude of Dielectric Absorption}

The magnitude of the dielectric absorption $\left(\varepsilon_{0}-\varepsilon_{\infty}\right)$ was obtained from the Cole-Cole plot. ${ }^{15}$ Here, $\varepsilon_{0}$ and $\varepsilon_{\infty}$ are the low- and high-frequency

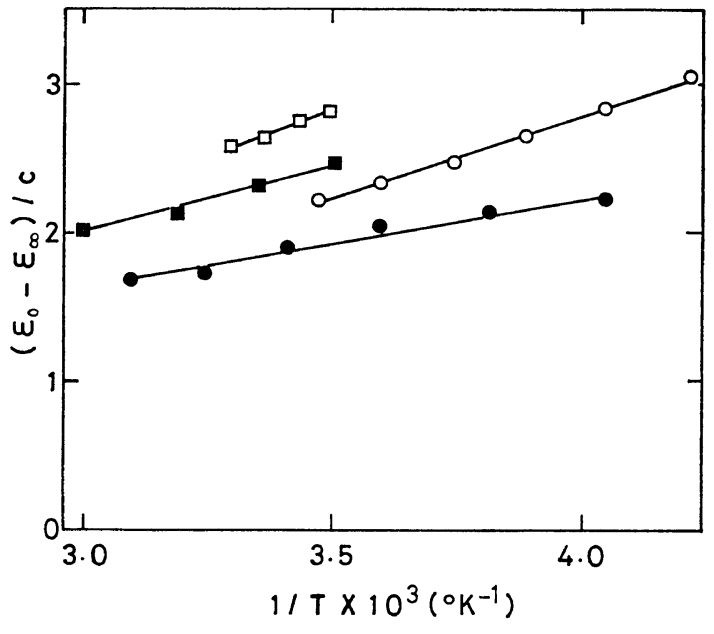

Figure 4. Temperature dependence of $\left(\varepsilon_{0}-\varepsilon_{\infty}\right) / c$ : $\bigcirc, 4.99 \mathrm{wt} \%$ I-PMMA in toluene; $\square, 2.02 \mathrm{wt} \%$ in dioxane; $4.90 \mathrm{wt} \%$ S-PMMA in toluene; $4.82 \mathrm{wt} \%$ in dioxane.

limits of the dielectric constant. The value of $\left(\varepsilon_{0}-\varepsilon_{\infty}\right)$ normalized by the concentration $c$ is plotted against the reciplocal of the absolute temperature $1 / T$ in Figure 4.

The value of $\left(\varepsilon_{0}-\varepsilon_{\infty}\right) / c$ becomes large in proportion to the increase of $1 / T$. It is found that the value of $\left(\varepsilon_{0}-\varepsilon_{\infty}\right) / c$ for I-PMMA is large in comparison with that for S-PMMA in the same solvent. Such a difference means that I-PMMA has a larger dipole moment than S-PMMA. ${ }^{16}$ This tendency is the same as that reported previously. ${ }^{7,8}$ It is worth noting the fact that the value of $\varepsilon_{\infty}$ obtained from the Cole-Cole plot for each solution is completely coincident with that of the solvent used. This fact means that no other relaxation processes exist at frequencies 


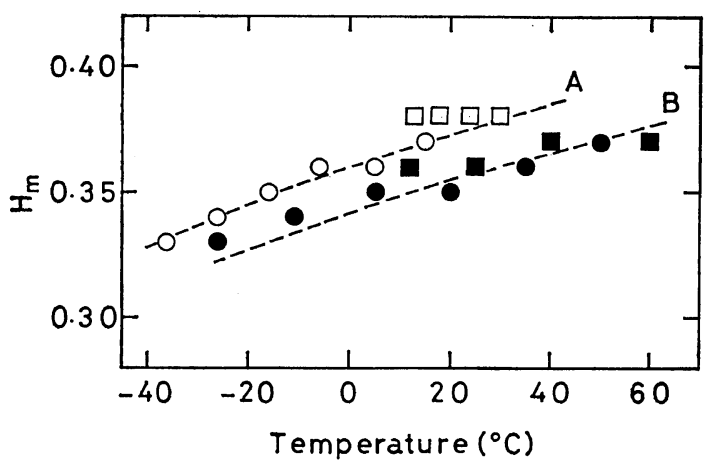

Figure 5. Temperature dependence of $H_{\mathrm{m}}$. Symbols are the same as in Figure 4. Dotted lines A and $\mathrm{B}$ represent the calculated values of $H_{\mathrm{m}}$ for 0.9 and $1.0 \mathrm{kcal} / \mathrm{mol}$ of $v_{0}$ respectively.

higher than those used in the present work.

\section{Distribution of Relaxation Times}

The shape of the dielectric loss curve was represented quantitatively using the distribution parameter $H_{\mathrm{m}}$ given by Kirkwood and Fuoss, ${ }^{17}$ where $H_{\mathrm{m}}$ is $\varepsilon_{\max }^{\prime \prime} /\left(\varepsilon_{0}-\varepsilon_{\infty}\right)$. The temperature dependence of $H_{\mathrm{m}}$ is shown in Figure 5. The value of $H_{\mathrm{m}}$ decreases slightly with decreasing temperature. No noticeable difference in the value of $H_{\mathrm{m}}$ between I- and S-PMMA can be observed.

\section{DISCUSSION}

It has been indicated that PMMA has flexible dipoles consisting of two kinds of components: one component is perpendicular to the direction of the main-chain, and the other is perpendicular to the first component. ${ }^{1}$ If the relaxation of the former component can occur independently of the latter, it will reflect the segmental motion of the main-chain just like those of vinyl-type polymers, so that it will be controlled by the solvent viscosity. ${ }^{9}$ On the other hand, the relaxation of the latter component will reflect the internal rotation of the side-chain in a relatively small region, which is supposed not to be much affected by the solvent viscosity. ${ }^{5}$

When each of the motions of main-chain and side-chain takes place independently of each other, one can expect to observe a dielectric loss curve which is dependent on the kind of solvent, or, in an extreme case, two peaks in the curve, because the two kinds of motions are affected in a different manner by the solvent viscosity. However, the value of $H_{\mathrm{m}}$ obtained for the toluene solution of PMMA is almost identical with that for the dioxane solution at the same temperature. This fact indicates that these two kinds of motions do not occur independently of each other, and implies that the dipole cannot be decomposed into two components.

\section{Dependence of Activation Energy and Relaxation} Time on Solvent Viscosity

Neither dielectric relaxation processes of Iand S-PMMA in solution is much dependent on the solvent viscosity, as shown in Tables II and III. This fact suggests that in dilute solution the dielectric relaxation mechanism of all kinds of PMMA, in spite of their different local conformations, are not to be interpreted in terms of the segmental motion of the main-chain. Their characteristic features may be explained in terms of a barrier model, where the relaxation time is determined by the time required to establish equilibrium among several states over potential barriers arising from molecular interactions.

Grigor'eva, et al. ${ }^{18}$ and Tanaka and Ishida ${ }^{19}$ showed from the analysis of the conformational energy that the strength of the intramolecular interactions between the main-chain and the side-chain of PMMA is sensitive to its stereoregularity. Their results also indicated that the rotation of the side-chain of I-PMMA occurs more easily than that of S-PMMA does. These conclusions suggest that a difference in the intramolecular interactions between I- and S-PMMA gives rise to differences in the dielectric activation energy and the relaxation time, in conformity with the experimental results. As already shown in Table II and III, I-PMMA in solution exhibits a smaller value of $\Delta H_{\mathrm{A}}$ and a shorter relaxation time than S-PMMA.

Ishida and Yamafuji ${ }^{2}$ treated in detail a chain model with four potential barriers, in which the rotations of main-chain and side-chain are both permitted; this gave successfully two relaxation times, corresponding to the so-called $\alpha$ - and $\beta$ processes. That model, however, cannot be utilized for the present case, since PMMA in 
solution shows only one relaxation. In this case, we cannot divide the potential barrier for the dipolar motion into two kinds of rotational motions, i.e., the main-chain and sidechain motions. Probably, the dipole jumps from one transient stable state to another over a single potential barrier. As described already, the observed relaxation process is not much concerned with the friction exerted from a viscous medium. Therefore, it seems to be reasonable to assume that the potential barrier is mainly governed by the intramolecular interactions between main-chain and side-chains. This view is supported by the analysis of the conformational energy. ${ }^{18,19}$ It will be important to introduce the energetic analysis method, which is available for the understanding of the sidechain motion in the glassy state, ${ }^{18,19}$ in order to predict exactly the existence of such a single potential barrier. However, this treatment is so complicated that we do not try it here.

Recently, it was reported that the dielectric relaxation of vinyl-type polymers can be well interpreted by the Kramers theory for large friction, qualitatively and also quantitatively. ${ }^{20,21}$ Nevertheless, an application of this theory to the dipolar rotation of PMMA in solution is inadequate, because the observed relaxation has no connection with the frictional resistance. Kramers also referred to the rate constant for the transition of particles over a potential barrier in another limiting case, that is, for sufficiently small friction. ${ }^{11}$

According to the Kramers theory for small friction, ${ }^{10,11}$ the rate constant $\omega$ is given by

$$
\omega=\frac{1}{2 \pi}\left(\frac{\gamma}{m}\right)^{1 / 2} \exp \left(-\frac{E^{*}}{k_{\mathrm{B}} T}\right)
$$

where $E^{*}$ is a height of a potential barrier, $m$ is the mass of a particle, and $k_{\mathrm{B}}$ is the Boltzmann constant. The parameter $\gamma$ is the curvature of a potential well near the position which the particles occupy first. Here, eq 1 contains no term related to the friction. This feature of eq 1 appears to be coincident with our above consideration for the dipolar rotation of PMMA in solution. If eq 1 is applied to the present case, $E^{*}$ should be replaced by $\Delta H_{\mathrm{A}}$. Furthermore, $m$ is replaced by the moment of inertia $I$ and $\gamma$ by $2 \Delta H_{\mathrm{A}} / N_{\mathrm{A}}$, which is derived from a two-states model, where $N_{\mathrm{A}}$ is Avogadro's number. The relaxation time $\tau$ for a two-states model is obtained by means of the barrier theory of Hoffman. ${ }^{22}$ When the rate constant for the transition of dipoles from state 1 to 2 is represented by $\omega_{12}$, and that from 2 to 1 by $\omega_{21}$, $\tau$ is given by

$$
\begin{aligned}
\tau & =1 /\left(\omega_{12}+\omega_{21}\right) \\
& =1 / \omega_{12}\left\{1+\exp \left(-\Delta H_{0} / k_{\mathrm{B}} T\right)\right\}
\end{aligned}
$$

where $\Delta H_{0}$ is the difference of the potential energy between that in state 1 and that in state 2. According to the Hoffman theory, in the case where a linear relationship is observed between $\left(\varepsilon_{0}-\varepsilon_{\infty}\right)$ and $1 / T$, as shown in Figure 4, the potential energies are equal in the two states, i.e., $\Delta H_{0}=0$. Then in the present case, $\tau$ is given by

$$
\tau=1 / 2 \omega_{12}
$$

Using eq 1 and 3, we obtain

$$
\tau=\pi\left(\frac{N_{\mathrm{A}} I}{2 \Delta H_{\mathrm{A}}}\right)^{1 / 2} \exp \left(\frac{\Delta H_{\mathrm{A}}}{R T}\right)
$$

where $R$ is the gas constant per mol. For the sake of simplicity, the value of $I$ is calculated only for the axis of a single side-chain in a monomer unit $\left(I=1 \times 10^{-38} \mathrm{~g} \mathrm{~cm}^{2}\right)$. In Table IV, the relaxation time $\tau_{\mathrm{c}}$ calculated from eq 4 is compared with $\tau_{e}$ obtained experimentally for I- and S-PMMA in toluene and dioxane at $288 \mathrm{~K}$. The agreement between the values of $\tau_{\mathfrak{c}}$ and $\tau_{\theta}$ is satisfactory. When the moment of inertia of a monomer around the main-chain is taken, the relaxation time estimated is only about twice as long as that calculated above. This suggests that the volume of the motional unit involved in the relaxation process is fairly small and nearly the same order as that of a monomer.

Table IV. Relaxation times of I- and S-PMMA in solutions at $288 \mathrm{~K}$

\begin{tabular}{lccc}
\hline Polymer & Solvent & $\tau_{\mathrm{c}}, \mathrm{sec}$ & $\tau_{\mathrm{e}}, \mathrm{sec}$ \\
\hline I-PMMA $^{\mathrm{a}}$ & Toluene & $2.8 \times 10^{-9}$ & $1.7 \times 10^{-9}$ \\
& Dioxane & $3.9 \times 10^{-9}$ & $2.8 \times 10^{-9}$ \\
S-PMMA $^{\mathrm{b}}$ & Toluene & $2.9 \times 10^{-8}$ & $7.1 \times 10^{-9}$ \\
& Dioxane & $2.9 \times 10^{-8}$ & $1.1 \times 10^{-8}$ \\
\hline
\end{tabular}

a Toluene, 4.99\%; dioxane, $2.02 \%$.

b Toluene, $4.90 \%$; dioxane, $4.82 \%$. 
As might be expected, such a small motional unit rotates without sweeping out a volume large enough to obey the Stokes law. This feature seems to satisfy the condition required for the application of eq 1 to our case.

\section{Distribution of Relaxation Times}

The result for the value of the parameter $H_{\mathrm{m}}$ indicates that the relaxation times distribute to the same extent in I- and S-PMMA in solutions at the given temperature. It was suggested that the dipolar rotation is controlled by the intramolecular interactions between main-chain and side-chains, which produce a single potential barrier. It is to be expected that there exist some fluctuations in the strength of the intramolecular interactions in solution, because polymer molecules in solutions take various conformations owing to the Brownian motion. It may be reasonable to suppose that such fluctuations yield a distribution of the barrier heights and give a broad spread of relaxation times.

Based on a barrier model, Fröhlich ${ }^{12}$ previously derived an equation for the relaxation time identical with eq 4 . Furthermore, he indicated that in a liquid or an amorphous solid, the potential barriers will vary in height, and considered that the energy barriers distribute with an uniform probability between $\Delta H_{\mathrm{A}}-v_{0}$ and $\Delta H_{\mathrm{A}}+v_{0}{ }^{12}$ This distribution model gives a symmetrical dielectric loss curve broader than that of a single relaxation process, which is dependent on temperature, ${ }^{12}$ such as observed in the present work. If we apply the Fröhlich consideration to eq $4, \Delta H_{\mathrm{A}}$ should be replaced by $\Delta H_{\mathrm{A}}+v$ in eq 4 , where $-v_{0} \leq v \leq v_{0}$. In the case of $v_{0} \neq 0$, the distribution of relaxation times is dependent not only on the exponential term in the right hand of eq 4 , but also on $\gamma$, i.e., $2\left(\Delta H_{\mathrm{A}}+v\right) / N_{\mathrm{A}}$. In comparison with the exponential term, however, $\gamma$ does not much contribute to the distribution, and may be taken as a constant. In this case, the Fröhlich equation for $v_{0} \neq 0$ also is identical with eq 4 . Then the relation of $H_{\mathrm{m}}$ and $v_{0}$ can be obtained: ${ }^{12,23}$

$$
\begin{aligned}
H_{\mathrm{m}}= & \frac{R T}{2 v_{0}}\left\{\tan ^{-1} \exp \left(\frac{v_{0}}{R T}\right)\right. \\
& \left.-\tan ^{-1} \exp \left(-\frac{v_{0}}{R T}\right)\right\}
\end{aligned}
$$

The values of $v_{0}$ giving the best fit for the values of $H_{\mathrm{m}}$ are found to be $0.9 \mathrm{kcal} / \mathrm{mol}$ for I-PMMA solutions and $1.0 \mathrm{kcal} / \mathrm{mol}$ for S-PMMA solutions. It is of interest to note that the temperature dependence of each calculated value of $H_{\mathrm{m}}$ is well fit with the experimental values over the temperature range examined, as shown in Figure 5. The Fröhlich model of the distribution of relaxation times seems to offer a good explanation for the dielectric loss curve broader than that of a single relaxation process. The above result may make our suggestion more plausible: that the potential barrier for the dipolar motion is caused by the intramolecular interactions between main-chain and side-chains in dilute solution of PMMA.

\section{CONCLUSION}

The values of dielectric activation energies obtained for I- and S-PMMA in dilute solution were not much affected by the solvent viscosity, but varied with the stereoregularity or, in other words, with the local conformation. The proportional relationships between the relaxation time and the solvent viscosity were not obtained in either case. These facts lead to the conclusion that, even in dilute solution, the dipolar motion of PMMA is mainly controlled by the intramolecular interactions. The dipolar rotation of PMMA is supposed to occur over a single potential barrier, the height of which is dependent on its local conformation. The relaxation times calculated by using the Kramers theory for small friction were in close agreement with the experimental ones. It is suggested that the volume of the motional unit involved in the relaxation process is fairly small. The shape of the dielectric loss curve observed for each solution and its temperature dependence can be well interpreted in terms of the Fröhlich distribution model. It is suggested that the distribution of the relaxation times should be attributed to some fluctuations in the strength of the intramolecular interactions.

\section{REFERENCES}

1. W. H. Stockmayer, Pure Appl. Chem., 15, 539 (1967). 
2. Y. Ishida and K. Yamafuji, Kolloid-Z., 177, 97 (1961).

3. M. G. McCrum, B. E. Read, and G. Williams, "Anelastic and Dielectric Effects in Polymeric Solids," John Wiley \& Sons, Inc., New York, N.Y., 1967, Chapter 8, pp 238-255.

4. A. M. North and P. J. Philips, Trans. Faraday Soc., 63, 1573 (1967).

5. S. Mashimo, A. Chiba, and K. Shinohara, Polymer J., 6, 170 (1974).

6. G. P. Mikhailov, A. M. Lobanov, and M.P. Platonov, Polym. Sci., USSR, 8, 1514 (1966).

7. H. A. Pohl, R. Bacskai, and W. P. Purcell, J. Phys. Chem., 64, 1701 (1960).

8. G. P. Mikhailov and L. L. Burshtein, Polym. Sci. USSR, 7, 957 (1965).

9. S. Mashimo and A. Chiba, Polymer J., 5, 41 (1973).

10. E. Helfand, J. Chem. Phys., 54, 4651 (1971).

11. H. A. Kramers, Physica, 7, 284 (1940).

12. H. Fröhlich, "Theory of Dielectrics," 2nd ed., Oxford University Press, London, 1958, pp 78, 90-98.

13. R. Bacskai and H. A. Poh1, J. Polym. Sci., 42,
151 (1960).

14. F. Bovey and G. V. D. Tiers, ibid., 44, 173 (1960).

15. K. S. Cole and R. H. Cole, J. Chem. Phys., 9, 341 (1941).

16. N. Hill, W. E. Vaughan, A. H. Price, and M. Davies, "Dielectric Properties \& Molecular Behaviour," Van Nortrand Reinhold Company, London, 1969, Chapter 4.

17. J. G. Kirkwood and R. M. Fuoss, J. Chem. Phys., 9, 329, (1941).

18. F. P. Grigor'eva and Yu. Ya. Gotlib, Polym. Sci. USSR, 10, 396 (1968).

19. A. Tanaka and Y. Ishida, J. Polym. Sci., Polym. Phys. Ed., 12, 335 (1974).

20. S. Mashimo, Y. Iwasa, A. Chiba, and K. Shinohara, J. Phys. Soc. Japan, 39, 1529 (1975).

21. S. Mashimo, Macromolecules, 9, 91 (1976).

22. J. D. Hoffman and H. G. Pfeiffer, J. Chem. Phys., 22, 132 (1954).

23. N. Hill, W. E. Vaugham, A. H. Price, and M. Davies, "Dielectric Properties \& Molecular Behaviour," Van Nostrand Reinhold Company, London, 1969, pp 84-88. 